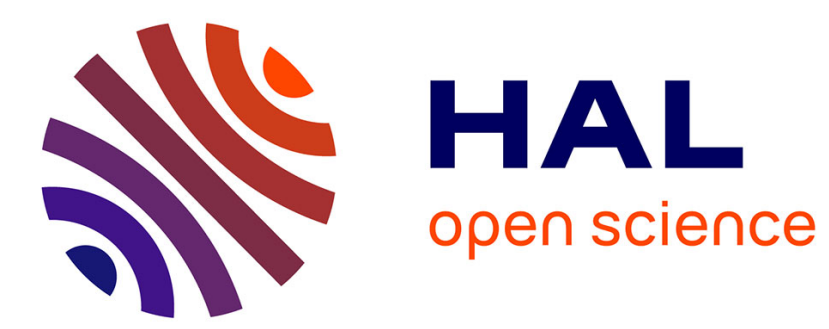

\title{
RADIAL INDEX RELATED TO AN INTERSECTION INDEX
}

Julie Lapebie

\section{- To cite this version:}

Julie Lapebie. RADIAL INDEX RELATED TO AN INTERSECTION INDEX. 2018. hal-01829125

\section{HAL Id: hal-01829125 \\ https://hal.science/hal-01829125}

Preprint submitted on 3 Jul 2018

HAL is a multi-disciplinary open access archive for the deposit and dissemination of scientific research documents, whether they are published or not. The documents may come from teaching and research institutions in France or abroad, or from public or private research centers.
L'archive ouverte pluridisciplinaire HAL, est destinée au dépôt et à la diffusion de documents scientifiques de niveau recherche, publiés ou non, émanant des établissements d'enseignement et de recherche français ou étrangers, des laboratoires publics ou privés. 


\title{
RADIAL INDEX RELATED TO AN INTERSECTION INDEX
}

\author{
JULIE LAPEBIE
}

\begin{abstract}
On a semialgebraic set $X$ with an isolated singularity at 0 , we are interested in relating the radial index of a vector field $V$ at the singularity to the one of $-V$. Then we write this radial index as an intersection index.
\end{abstract}

\section{INTRODUCTION}

To work on manifolds with singularities, we cannot work with the Poincaré-Hopf index, which is only defined on smooth manifolds, we need to work with another one, the radial index (or Schwartz index depend on the authors). This index was introduced by Marie-Hélène Schwartz ([11], [12]) but only concerned radial vector fields. Later, a generalization to other vector fields was made by Henry King and David trotman in [10] and independently by [1], [5] and [13]. We will use the definition of [6].

On a semialgebraic set $X$ of dimension $k$ in $\mathbb{R}^{N}$ with an isolated singularity at 0 , we consider a vector field $V$. We want to write the radial index of $V$ at 0 in terms of the zeros of $V_{l l k(X)}$, the restriction of $V$ to the link of the singularity (Proposition 2.5). Then we relate, in Theorem 2.8, the radial index of $V$ at 0 to the one of $-V$ at 0 :

- if $k$ is even, then

$$
\operatorname{ind}_{\text {rad }}(-V, 0)=i n d_{r a d}(V, 0)
$$

- if $k$ is odd, then

$$
i n d_{r a d}(V, 0)+i n d_{r a d}(-V, 0)=2-\chi(l k(X)) .
$$

It follows the corollary:

$$
i n d_{\text {rad }}(V, 0)+i n d_{\text {rad }}(-V, 0) \equiv \chi(l k(X)) \bmod 2 .
$$

In the case where we have an analytic map $f:\left(\mathbb{R}^{n}, 0\right) \rightarrow\left(\mathbb{R}^{k}, 0\right)$ defined on a neighborhood of 0 and such that the fiber $f^{-1}(0)$ admits an isolated singularity, Giorgi Khimshiashvili ([9]) proved the following formula:

$$
\chi\left(f^{-1}(\delta) \cap B_{\varepsilon}^{n}\right)=1-\operatorname{sign}(-\delta)^{n} \operatorname{deg}_{0} \nabla f,
$$

where $\delta$ is a regular value of $f, \operatorname{deg}_{0} \nabla f$ the local degree of $\frac{\nabla f}{\|\nabla f\|}: S_{\varepsilon}^{n-1} \rightarrow S^{n-1}$ and $0<\delta \ll \varepsilon \ll 1$.

From Theorem 2.8, we obtain a singular version of Khimshiashvili's formula:

Let $g: X \rightarrow \mathbb{R}$ be the restriction to $X$ of a $\mathcal{C}^{2}$-semialgebraic function without any critical points on a neighborhood of 0 and such that $g(0)=0$. Then

2010 Mathematics Subject Classification. 14P10, 14P25, 58K05.

Key words and phrases. Radial index, semialgebraic set, Euler characteristic. 
- if $k$ is even,

$$
\chi\left(g^{-1}(\delta) \cap B_{\varepsilon}^{N}\right)=1-i n d_{r a d}(\nabla g, 0) ;
$$

- if $k$ is odd,

$$
\chi\left(g^{-1}(\delta) \cap B_{\varepsilon}^{N}\right)=\chi(l k(X))-1+i n d_{r a d}(\nabla g, 0),
$$

where $\delta$ is a regular value of $g$.

This gives us a direct connection between the Euler characteristic of the Milnor fiber of $g$ and the radial index of $\nabla g$ at 0 .

Finally, we remark that a coincidence point $x \in l k(X)$ of $V$ and $-\nabla \rho$, where $\nabla \rho$ is a radial vector field, is an inward zero of $V_{\mid l k(X)}$. Then, we would like to relate the radial index of $V$ at 0 to an intersection index with the radial vector field $\nabla \rho$. For this, we consider the normalized vector fields $\widehat{V}$ and $-\nabla \widehat{\rho}$ on $l k(X)$ and their respective graphs $G_{\widehat{V}}$ and $G_{-\nabla \widehat{\rho}}$. In Proposition 3.3, we prove that:

$$
I\left(G_{-\nabla \hat{\rho}}, G_{\hat{V}}\right)=1-i n d_{\text {rad }}(V, 0),
$$

where $I\left(G_{-\nabla \hat{\rho}}, G_{\hat{V}}\right)$ is the intersection index of the graphs.

The paper is organized as follows: in Section 2, we write the radial index of $V$ at 0 as a sum involving only the zeros of $V_{l l k(X)}$. This enables us to prove Theorem 2.8 and the two corollaries presented above. Section 3 relates the radial index of $V$ at 0 to an intersection index of the graph of $V$ with the graph of the opposite of a radial vector field.

\section{About the RADIAL INDEX}

Let us consider a $k$-dimensional semialgebraic set $X \subset \mathbb{R}^{N}$ with an isolated singularity at 0 .

We denote by $B_{\varepsilon}^{N}$ the closed ball of dimension $N$, radius $\varepsilon$ and centered at $0, S_{\varepsilon}^{N-1}=$ $\partial B_{\varepsilon}^{N}$ the sphere of dimension $N-1$ and $\stackrel{\circ}{B}_{\varepsilon}^{N}=B_{\varepsilon}^{N} \backslash S_{\varepsilon}^{N-1}$. We denote by $l k(X)$ the link of $X$ at 0 , i.e. the intersection of $X$ with $S_{\varepsilon}^{N-1}$ where $\varepsilon>0$ is such that 0 is the only singularity of $X$ in $X \cap B_{\varepsilon}^{N}$ and $0<\varepsilon \ll 1$.

Before speaking about the radial index of a vector field at a singularity, we need to define what is a vector field at a singularity and what is a radial vector field.

Definition 2.1. [3] p.32.

Let $V$ be a continuous vector field on $X \subset \mathbb{R}^{N}$. We say that $V$ admits an isolated singularity at $0 \in X$ if $V$ is a continuous section of $T \mathbb{R}_{\mid X}^{N}$ tangent to $X \backslash\{0\}$ and does not have any zeros on a small neighborhood of 0 .

Definition 2.2. A continuous vector field $V_{\text {rad }}$ tangent to $X$ is called radial (at 0 ) if it is transverse to the link of $X$. Moreover, we make the convention that it is pointing inward, i.e. it is not pointing toward 0. A zero of $V_{\text {rad }}$ is called an inward zero (resp. an outward zero) if $V_{\text {rad }}$ is pointing inward (resp. outward) at that point. 
Definition 2.3. [7] Definition 2.1, [5], [6] section 1 and [3] Chapter 2.

Let us consider a continuous vector field $V$ on $X$ with a singularity at 0 .

Let $\varepsilon>0$ be such that $V$ does not have any zeros on $X \backslash\{0\} \cap B_{\varepsilon}^{N}$ and let $0<\varepsilon^{\prime}<\varepsilon$. Let us denote by $X_{\varepsilon \varepsilon^{\prime}}=\left(X \cap B_{\varepsilon}^{N}\right) \backslash \stackrel{\circ}{B}_{\varepsilon^{\prime}}^{N}$ and by $\partial X_{\varepsilon \varepsilon^{\prime}}=X_{\varepsilon} \cup X_{\varepsilon^{\prime}}$ with $X_{\varepsilon}=X \cap S_{\varepsilon}^{N-1}$ and $X_{\varepsilon^{\prime}}=X \cap S_{\varepsilon^{\prime}}^{N-1}$.

Let $\widetilde{V}$ be a vector field on $X_{\varepsilon \varepsilon^{\prime}}$ such that:

- $\widetilde{V}_{\mid X_{\varepsilon}}=V$;

- $\widetilde{V}_{\mid X_{\varepsilon^{\prime}}}=V_{\text {rad }}$ is a radial vector field;

- $\widetilde{V}_{\mid \dot{X}_{\varepsilon \varepsilon^{\prime}}}$ has a finite number of zeros $\left(p_{i}\right)_{1 \leq i \leq s}$ and they are all nondegenerate.

Then we define the radial index of $V$ at 0 , which we write ind rad $(V, 0)$, as:

$$
\operatorname{ind}_{\text {rad }}(V, 0):=1+\sum_{i=1}^{s} \operatorname{ind}_{P H}\left(\widetilde{V}, p_{i}\right)
$$

For the next proposition, we will need to define a correct zero of a vector field.

Definition 2.4. We say that a zero $p \in l k(X)$ of $V$ is correct if it is just a zero of $V_{\mid l k(X)}$ and not of $V$ on $X$.

Proposition 2.5. Let $X$ be a semialgebraic closed set with 0 as an isolated singularity and $V$ a vector field defined on a neighborhood $\mathcal{U}_{0}$ of 0 in $X$. Let $\mathcal{U}_{1} \varsubsetneqq \mathcal{U}_{0}$ be a neighborhood of $l k(X)$. Let $\widetilde{V}$ be a $\mathcal{C}^{1}$-perturbation of $V$ on $\mathcal{U}_{0}$ such that $\widetilde{V}_{\mid l k(X)}$ admits a finite number of isolated nondegenerate zeros $r_{1}, \ldots, r_{t}$ which are all correct; and such that $\widetilde{V}$ is equal to $V$ on $\mathcal{U}_{0} \backslash \mathcal{U}_{1}$. Then we have the equality

$$
\operatorname{ind}_{\text {rad }}(V, 0)=1-\sum_{\substack{i=1 \\ r_{i} \text { inward zero }}}^{t} \operatorname{ind}_{P H}\left(\widetilde{V}_{\mid l k(X)}, r_{i}\right)
$$

Remark 2.6. This proposition is a particular case of Definition 5.5 in [10].

Proof. Let $0<\varepsilon^{\prime}<\varepsilon$. Let us keep the notations of Definition 2.3. Let $\widetilde{\widetilde{V}}$ a perturbation of $\widetilde{V}$ on $\mathcal{U}_{0}$ such that:

- $\widetilde{\widetilde{V}}_{\mid \dot{X}_{\varepsilon \varepsilon^{\prime}}}$ admits a finite number of nondegenerate zeros $p_{1}, \ldots, p_{s}$;

- $\widetilde{\widetilde{V}}_{\mid X_{\varepsilon}}=\widetilde{V}_{\mid X_{\varepsilon}}=\widetilde{V}_{\mid l k(X)}$,

- $\widetilde{\widetilde{V}}_{\mid X_{\varepsilon^{\prime}}}$ is a $\mathcal{C}^{1}$-perturbation of $V_{\text {rad }}$ on $X_{\varepsilon^{\prime}}$ having only nondegenerate zeros $q_{1}, \ldots, q_{u}$ which are all inward in $X_{\varepsilon \varepsilon^{\prime}}$ (for $V_{\text {rad }}$ is radial so is pointing inward at every point of $X_{\varepsilon^{\prime}}$ ). 
Applying Poincaré-Hopf theorem for manifold with boundary (see for example [2] or [4]) to the vector field $\widetilde{\widetilde{V}}$ on the set $X_{\varepsilon \varepsilon^{\prime}}$, we get the equality:

$$
\begin{aligned}
\chi\left(X_{\varepsilon \varepsilon^{\prime}}\right) & =\sum_{i=1}^{s} i n d_{P H}\left(\widetilde{\widetilde{V}}, p_{i}\right)+\sum_{\substack{i=1 \\
q_{i} \text { inward in } X_{\varepsilon \varepsilon^{\prime}}}}^{u} \operatorname{ind}_{P H}\left(\widetilde{\widetilde{V}}_{\mid X_{\varepsilon^{\prime}}}, q_{i}\right) \\
& +\sum_{\substack{i=1 \\
r_{i} \text { inward in } X_{\varepsilon \varepsilon^{\prime}}}}^{t} \operatorname{ind}_{P H}\left(\widetilde{V}_{\mid X_{\varepsilon}}, r_{i}\right) .
\end{aligned}
$$

As $\widetilde{\widetilde{V}}_{\mid X_{\varepsilon^{\prime}}}$ only admits inward zeros on $X_{\varepsilon^{\prime}}$, we have

$$
\chi\left(X_{\varepsilon^{\prime}}\right)=\sum_{i=1}^{u} \operatorname{ind} d_{P H}\left(\left.\widetilde{\widetilde{V}}\right|_{X_{\varepsilon^{\prime}}}, q_{i}\right)
$$

and so

$$
\chi\left(X_{\varepsilon \varepsilon^{\prime}}\right)-\chi\left(X_{\varepsilon^{\prime}}\right)=\sum_{i=1}^{s} \operatorname{ind}_{P H}\left(\widetilde{\widetilde{V}}, p_{i}\right)+\sum_{\substack{i=1 \\ r_{i} \text { inward in } X_{\varepsilon \varepsilon^{\prime}}}}^{t} \operatorname{ind}_{P H}\left(\left.\widetilde{V}\right|_{X_{\varepsilon}}, r_{i}\right) .
$$

But $X_{\varepsilon^{\prime}}$ is a retract by deformation of $X_{\varepsilon \varepsilon^{\prime}}$ so $\chi\left(X_{\varepsilon \varepsilon^{\prime}}\right)=\chi\left(X_{\varepsilon^{\prime}}\right)$. Then we find

$$
\sum_{i=1}^{s} i n d_{P H}\left(\widetilde{\widetilde{V}}, p_{i}\right)=-\sum_{\substack{i=1 \\ r_{i} \text { inward in } X_{\varepsilon \varepsilon^{\prime}}}}^{t} i n d_{P H}\left(\left.\widetilde{V}\right|_{X_{\varepsilon}}, r_{i}\right),
$$

and therefore,

$$
\underbrace{1+\sum_{i=1}^{s} i n d_{P H}\left(\widetilde{V}, p_{i}\right)}_{=i n d_{\text {rad }}(V, 0)}=1-\sum_{\substack{i=1 \\ r_{i} \text { inward in } X_{\varepsilon \varepsilon^{\prime}}}}^{t} \operatorname{ind}_{P H}\left(\left.\widetilde{V}\right|_{X_{\varepsilon}}, r_{i}\right),
$$

which gives us the required result.

Remark 2.7. The proof of the next theorem is similar to the one of Poincaré-Hopf theorem for manifolds with boundary.

Theorem 2.8. Let $X$ be a closed semialgebraic set of dimension $k$ with 0 as an isolated singularity and let $V$ be a vector field on $X$ with a singularity at 0 . Then we have the following equalities:

- if $k$ is even, then

$$
i n d_{r a d}(-V, 0)=i n d_{r a d}(V, 0)
$$

- if $k$ is odd, then

$$
i n d_{r a d}(V, 0)+i n d_{r a d}(-V, 0)=2-\chi(l k(X)) .
$$


Proof. Let $\widetilde{V}$ be a $\mathcal{C}^{1}$-perturbation of $V$ satisfying conditions of Proposition 2.5. We denote by $\left(q_{i}\right)_{1 \leq i \leq t}$ the inward zeros of $\widetilde{V}$ and $\left(p_{i}\right)_{1 \leq i \leq s}$ the outward zeros. By Proposition 2.5 , we can write

$$
i n d_{\text {rad }}(-V, 0)=1-\sum_{r \text { inward zero of }-\left.\widetilde{V}\right|_{l k(X)}} i n d_{P H}\left(-\left.\widetilde{V}\right|_{l k(X)}, r\right),
$$

and

$$
\sum_{r \text { inward zero of }-\left.\widetilde{V}\right|_{l k(X)}} i n d_{P H}\left(-\left.\widetilde{V}\right|_{l k(X)}, r\right)=\sum_{i=1}^{s} i n d_{P H}\left(-\left.\widetilde{V}\right|_{l k(X)}, p_{i}\right) .
$$

As $X$ has dimension $k$, the link $l k(X)$ has dimension $k-1$ and so

$$
\operatorname{ind}_{P H}\left(-\left.\widetilde{V}\right|_{l k(X)}, p_{i}\right)=(-1)^{k-1} i n d_{P H}\left(\left.\widetilde{V}\right|_{l k(X)}, p_{i}\right) .
$$

Moreover,

$$
\begin{aligned}
\operatorname{ind}_{\text {rad }}(V, 0) & =1-\sum_{r \text { inward zero of }\left.\tilde{V}\right|_{l k(X)}} \operatorname{ind}_{P H}\left(\left.\widetilde{V}\right|_{l k(X)}, r\right) \\
& =1-\sum_{i=1}^{t} i n d_{P H}\left(\left.\widetilde{V}\right|_{l k(X)}, q_{i}\right) .
\end{aligned}
$$

Thus,

$$
\begin{aligned}
S & :=\operatorname{ind}_{\text {rad }}(V, 0)+i n d_{r a d}(-V, 0) \\
& =2-\sum_{i=1}^{t} i n d_{P H}\left(\left.\widetilde{V}\right|_{l k(X)}, q_{i}\right)-(-1)^{k-1} \sum_{i=1}^{s} i n d_{P H}\left(\left.\widetilde{V}\right|_{l k(X)}, p_{i}\right) .
\end{aligned}
$$

We also have the equality

$$
\chi(l k(X))=\sum_{i=1}^{t} \operatorname{ind}_{P H}\left(\left.\widetilde{V}\right|_{l k(X)}, q_{i}\right)+\sum_{i=1}^{s} i n d_{P H}\left(\left.\widetilde{V}\right|_{l k(X)}, p_{i}\right) .
$$

Let us study the result according to the parity of $k$.

- If $k$ is even, then

$$
\begin{aligned}
S & =2-\sum_{i=1}^{t} i n d_{P H}\left(\widetilde{V}_{l l k(X)}, q_{i}\right)+\sum_{i=1}^{s} i n d_{P H}\left(\widetilde{V}_{l k k(X)}, p_{i}\right) \\
& =2-\sum_{i=1}^{t} i n d_{P H}\left(\widetilde{V}_{l l k(X)}, q_{i}\right)+\chi(l k(X))-\sum_{i=1}^{t} i n d_{P H}\left(\widetilde{V}_{l l k(X)}, q_{i}\right) \\
& =2-2 \sum_{i=1}^{t} i n d_{P H}\left(\widetilde{V}_{l l k(X)}, q_{i}\right)+\chi(l k(X)) \\
& =2 i n d_{r a d}(V, 0)+\chi(l k(X)) .
\end{aligned}
$$


As dimension of $l k(X)$ is odd (equal to $k-1$ ), its Euler characteristic vanishes. Therefore

$$
i n d_{r a d}(-V, 0)=i n d_{r a d}(V, 0)
$$

- If $k$ is odd, then

$$
\begin{aligned}
S & =2-\sum_{i=1}^{t} i n d_{P H}\left(\widetilde{V}_{\mid l k(X)}, q_{i}\right)-\sum_{i=1}^{s} i n d_{P H}\left(\widetilde{V}_{\mid l k(X)}, p_{i}\right) \\
& =2-\chi(l k(X)) .
\end{aligned}
$$

Once we have proved this theorem, the following result is straightforward:

Corollary 2.9. Let $X$ be a closed semialgebraic set with an isolated singularity at 0 and let $V$ be a vector field on $X$ with a singularity at 0 . Then

$$
i n d_{r a d}(V, 0)+i n d_{r a d}(-V, 0) \equiv \chi(l k(X)) \bmod 2 .
$$

Proof. In the previous theorem, we have seen that if $k$ is even, then

$$
S:=\operatorname{ind}_{\text {rad }}(V, 0)+i n d_{\text {rad }}(-V, 0)=2 \operatorname{ind}_{\text {rad }}(V, 0) \equiv 0 \bmod 2,
$$

and in that case $\chi(l k(X))=0$, so

$$
S \equiv \chi(l k(X)) \quad \bmod 2
$$

If $k$ is odd, then

$$
S=2-\chi(l k(X))
$$

and hence

$$
S \equiv \chi(l k(X)) \quad \bmod 2
$$

Finally, we have the following corollary, which is a singular version of Khimshiashvili's formula:

Corollary 2.10. Let $X \subset \mathbb{R}^{N}$ a closed semialgebraic set of dimension $k$ with an isolated singularity at 0 and let us consider a function $g: X \rightarrow \mathbb{R}$ restriction to $X$ of a $\mathcal{C}^{2}$-semialgebraic function. Let us suppose that $g(0)=0$ and that $g$ does not have any critical points on a neighborhood of 0 . Let $\delta$ be a regular value of $g$ and $\varepsilon>0$ such that $0<\delta \ll \varepsilon \ll 1$. Then we have the following equalities:

- if $k$ is even,

$$
\chi\left(g^{-1}(\delta) \cap B_{\varepsilon}^{N}\right)=1-i n d_{r a d}(\nabla g, 0) ;
$$

- if $k$ is odd,

$$
\chi\left(g^{-1}(\delta) \cap B_{\varepsilon}^{N}\right)=\chi(l k(X))-1+i n d_{r a d}(\nabla g, 0) .
$$


Proof. From [7] Example 2.6, we know that for $0<\delta \ll \varepsilon \ll 1$, we have

$$
\chi\left(g^{-1}(-\delta) \cap B_{\varepsilon}^{N}\right)=1-i n d_{r a d}(\nabla g, 0) .
$$

So

$$
\chi\left(g^{-1}(\delta) \cap B_{\varepsilon}^{N}\right)=1-i n d_{r a d}(-\nabla g, 0) .
$$

Thus, from the previous theorem,

- if $k$ is even, then

$$
i n d_{\text {rad }}(-\nabla g, 0)=i n d_{\text {rad }}(\nabla g, 0)
$$

and so

$$
\chi\left(g^{-1}(\delta) \cap B_{\varepsilon}^{N}\right)=1-i n d_{r a d}(\nabla g, 0) ;
$$

- if $k$ is odd, then

$$
i n d_{\text {rad }}(-\nabla g, 0)=2-\chi(l k(X))-i n d_{\text {rad }}(\nabla g, 0)
$$

and therefore

$$
\begin{aligned}
\chi\left(g^{-1}(\delta) \cap B_{\varepsilon}^{N}\right) & =1-i n d_{r a d}(-\nabla g, 0) \\
& =\chi(l k(X))-1+i n d_{r a d}(\nabla g, 0) .
\end{aligned}
$$

\section{A LINK WITH AN INTERSECTION INDEX}

First, we recall the definition of the intersection index of two manifolds [8].

Definition 3.1. Let $X, Y$ and $Z$ be three oriented manifolds without boundary such that $X, Z \subset Y$. We suppose $X$ compact, $Z$ closed and $\operatorname{dim} X+\operatorname{dim} Z=\operatorname{dim} Y$.

If $X$ and $Z$ are transverse, then we define the intersection index $I(X, Z)$ of $X$ and $Z$ counting the points $x$ of the intersection $X \cap Z$ and associating to each a plus sign + or a minus sign -. More precisely, if for $x \in X \cap Z$ the given orientation of $T_{x} X \times T_{x} Z$ (where we denoted by $T_{x} X$ the tangent plane of $X$ at $x$ ) is the same as the one given by the tangent plane $T_{x} Y$, then we associate a plus sign + to $x$. We set or $(x)=1$. Otherwise, we associate a minus sign - and we set or $(x)=-1$. Let $C(X, Z)$ be the set of intersection points of $X$ and $Z$. Then we have the equality

$$
I(X, Z):=\sum_{x \in C(X, Z)} \operatorname{or}(x)
$$

Remark 3.2. When $X$ and $Z$ are not transverse, we perturb $X$ and $Z$ in $\tilde{X}$ and $\tilde{Z}$ respectively so that $\tilde{X}$ and $\tilde{Z}$ are. Then the intersection index $I(\tilde{X}, \tilde{Z})$ exists according to the previous definition. Moreover, as this index is invariant under perturbations, we define $I(X, Z)$ as $I(X, Z)=I(\tilde{X}, \tilde{Z})$.

Let $X \subset \mathbb{R}^{N}$ be a semialgebraic variety of dimension $k+1$ with an isolated singularity at 0 . We suppose that $X \backslash\{0\}$ is oriented. For example, we can consider a set of the form $X=\left\{f_{1}=\ldots=f_{l}=0\right\}$ such that $\operatorname{rank}\left(\nabla f_{1}(x), \ldots, \nabla f_{l}(x)\right)=0$ for all $x \in X \backslash\{0\}$.

We consider a $\mathcal{C}^{2}$-tangent vector field $V$ on $X \backslash\{0\}$ and $\nabla \rho$ a $\mathcal{C}^{2}$-radial vector field on $X$. Then we consider the link of $X$ at 0 , which we denote by $l k(X)$, i.e. the 


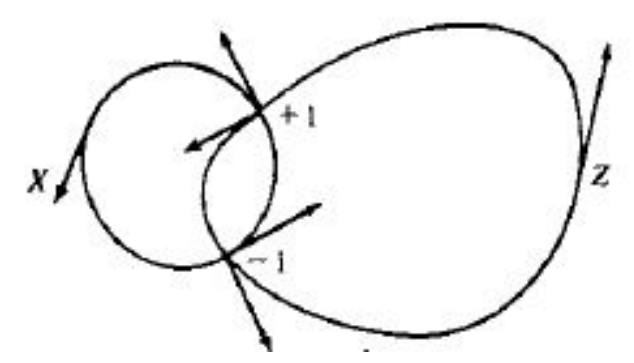

FiguRE 1. Example in dimension 2.

intersection of $X$ with $S_{\varepsilon}^{N-1}$ where $\varepsilon>0$ is such that 0 is the only singularity of $X$ in $X \cap B_{\varepsilon}^{N}$ and $0<\varepsilon \ll 1$.

The vector field $V$ does not admit any zero on $l k(X)$. For a care of simplicity, we set $Y:=l k(X)$. We can remark that $Y$ is an oriented submanifold of $X$ of dimension $k$. Indeed, we know that there exists a $(k+1)$-dimensional manifold with boundary $Z$ such that the boundary $\partial Z$ of $Z$ is equal to $Y$. Here, $Z=X \cap B_{\varepsilon}^{N} \backslash\{0\}$. We recall that the orientation of $Y$ is given by the one of $Z$. Let $\left(v_{1}, \ldots, v_{k}\right)$ be a basis of $Y=\partial Z$ and let $x \in Y$. The space $T_{x} Y$ has codimension 1 in $T_{x} Z$ so there exist exactly two unit vectors $\vec{v}$ and $-\vec{v}$ in $T_{x} Z$ which are perpendicular to $T_{x} Y, \vec{v}$ pointing outward and $-\vec{v}$ inward. We suppose that $\left(v_{1}, \ldots, v_{k}, \vec{v}\right)$ is a positively oriented basis of $T_{x} Z$. Then the basis $\left(v_{1}, \ldots, v_{k}\right)$ is positively oriented for $T_{x} Y$ and so is for $Y$.

For all $x \in Y$, we introduce these two vector fields:

$$
\widehat{V}(x)=\frac{V(x)}{\|V(x)\|} \text { and }-\nabla \widehat{\rho}(x)=-\frac{\nabla \rho(x)}{\|\nabla \rho(x)\|} .
$$

We say that $x$ is a coincidence point of $\widehat{V}$ and $-\nabla \widehat{\rho}$ if $\widehat{V}(x)=-\nabla \widehat{\rho}(x)$. We want to relate these coincidence points to the inward zeros of the vector field $V_{\mid Y}$. Indeed,

$$
\begin{aligned}
\widehat{V}(x)=-\nabla \widehat{\rho}(x) & \Longleftrightarrow V(x)=-\frac{\|V(x)\|}{\|\nabla \rho(x)\|} \nabla \rho(x) \\
& \Longleftrightarrow x \text { is an inward zero of } V_{\mid Y} .
\end{aligned}
$$

Let us introduce the following set:

$$
T U Y=\left\{(x, \vec{v}) \mid x \in Y, \vec{v} \in T_{x} X \text { and }\|\vec{v}\|=1\right\},
$$

which is included in the unit tangent bundle of $X$, that is

$$
T U X=\left\{(x, \vec{v}) \mid x \in X, \vec{v} \in T_{x} X \text { and }\|\vec{v}\|=1\right\} .
$$

The bundle $T U X$ is locally the product of an open set in $\mathbb{R}^{k}$ with the $k$-sphere $S^{k}$ so has dimension $\operatorname{dim} X+\operatorname{dim} S^{k}=k+1+k=2 k+1$.

The set $T U Y$ is an oriented manifold of dimension $2 k$.

Indeed, $T U Y=T U X \cap\left(\{\rho(x)=\varepsilon\} \times \mathbb{R}^{k+1}\right)$. The tangent bundle $T X$ of $X$ is oriented so is $T U X$. Actually, $T U X$ is the transverse intersection of $T X$ with $\mathbb{R}^{n} \times\{\vec{v} \mid\|\vec{v}\|=1\}$, that is also an oriented manifold. As the two manifolds $T U X$ and 
$\{\rho(x)=\varepsilon\} \times \mathbb{R}^{k+1}$ are oriented and that their intersection is transverse, TUY is also oriented. Moreover, its dimension is equal to $2 k$ for it is equal to $\operatorname{dim} T U X-1$.

We consider $G_{\widehat{V}}$ and $G_{-\nabla \widehat{\rho}}$ the respective graphs of $\widehat{V}$ and $-\nabla \widehat{\rho}$ :

$$
G_{\widehat{V}}=\{(x, \widehat{V}(x)) \mid x \in Y\} \text { and } G_{-\nabla \widehat{\rho}}=\{(x,-\nabla \widehat{\rho}(x)) \mid x \in Y\} .
$$

These graphs are oriented subsets of $T U Y$. A positively oriented basis of $T_{(x, \widehat{V}(x))} G_{\widehat{V}}$ is of the form $\left\langle\left(\varepsilon_{1}, D \widehat{V}(x)\left(\varepsilon_{1}\right)\right), \ldots,\left(\varepsilon_{k}, D \widehat{V}(x)\left(\varepsilon_{k}\right)\right)\right\rangle$ for $\left(\varepsilon_{1}, \ldots, \varepsilon_{k}\right)$ is a positively oriented basis of $Y$.

Then we prove the following result:

Proposition 3.3. Let $X \subset \mathbb{R}^{N}$ be a semialgebraic set of dimension $k+1$ with an isolated singularity at 0 and such that $X \backslash\{0\}$ is oriented. Let $V$ be a $\mathcal{C}^{2}$-vector field and $\nabla \rho$ a $\mathcal{C}^{2}$-radial vector field on $X$, and let us consider their respective normalized vector field $\widehat{V}$ and $\nabla \widehat{\rho}$ defined on the link of $X$ at the singularity. Then we have the equality:

$$
I\left(G_{-\nabla \widehat{\rho}}, G_{\widehat{V}}\right)=1-i n d_{r a d}(V, 0) .
$$

Proof. The graphs $G_{\widehat{V}}$ and $G_{-\nabla \widehat{\rho}}$ of $\widehat{V}$ and $-\nabla \widehat{\rho}$ respectively are oriented compact submanifolds of $T U Y$, each of dimension $k$. Then their intersection index $I\left(G_{-\nabla \widehat{\rho}}, G_{\widehat{V}}\right)$ is well defined.

Let us consider a coincidence point $x \in Y(=l k(X))$ of $\widehat{V}$ and $-\nabla \widehat{\rho}$. The point $(x, \widehat{V}(x))$ is in that case an intersection point of the two graphs $G_{\widehat{V}}$ and $G_{-\nabla \widehat{\rho}}$. We are going to compute the value of $\operatorname{or}((x, \widehat{V}(x)))$, which we denote for simplicity by $\operatorname{or}(x)$, to relate it with $\operatorname{ind}_{P H}\left(V_{\mid Y}, x\right)$, the Poincaré-Hopf index of $V_{\mid Y}$ at $x$.

To compute or $(x)$, we must find a positively oriented basis of the tangent plane to $T U Y$ at $(x, \widehat{V}(x))$ and one for the two tangent planes $T_{(x, \widehat{V}(x))} G_{\widehat{V}}$ and $T_{(x, \widehat{V}(x))} G_{-\nabla \widehat{\rho}}$.

Let us begin with $T_{(x, \widehat{V}(x))} T U Y$.

As $X \backslash\{0\}$ is a manifold of dimension $k+1$, we know that there exists a neighborhood $\mathcal{U}_{1}$ of $x \in Y$ in $X$, and a diffeomorphism $\phi_{1}$ such that $\phi_{1}\left(\mathcal{U}_{1}\right)$ is an open set of $\mathbb{R}^{k+1}$. Moreover, $Y$ is a submanifold of $X$ of dimension $k$ so $\mathcal{U}_{1} \cap Y$ is a neighborhood of $x$ in $Y$ and $\phi_{1}\left(\mathcal{U}_{1} \cap Y\right)=\phi_{1}\left(\mathcal{U}_{1}\right) \cap \mathbb{R}^{k}$ is an open set of $\mathbb{R}^{k}$, where we identify $\mathbb{R}^{k}$ with $\mathbb{R}^{k} \times\{0\} \subset \mathbb{R}^{k+1}$. We will write $\mathbb{R}^{k} \times\{0\}=\left\{x_{k+1}=0\right\}$. So

$$
\phi_{1}\left(\mathcal{U}_{1} \cap Y\right)=\phi_{1}\left(\mathcal{U}_{1}\right) \cap\left\{x_{k+1}=0\right\} .
$$

Moreover, as $Z$ is a manifold with boundary included in $X$ of dimension $k+1$ and such that $\partial Z=Y$, the open set $\mathcal{U}_{1} \cap Z$ is a neighborhood of $x$ in $Z$ and

$$
\phi_{1}\left(\mathcal{U}_{1} \cap Z\right)=\phi_{1}\left(\mathcal{U}_{1}\right) \cap\left\{x_{k+1} \geq 0\right\},
$$

which is an open set of $\mathbb{R}^{k} \times \mathbb{R}_{+}$(where we wrote $\left\{x_{k+1} \geq 0\right\}=\mathbb{R}^{k} \times \mathbb{R}_{+}$).

Locally, if $x \in \mathcal{U}_{1}$, the bundle $T X$ is diffeomorphic to $\phi_{1}\left(\mathcal{U}_{1}\right) \times \mathbb{R}^{k+1}$ and $T U X$ to $\phi_{1}\left(\mathcal{U}_{1}\right) \times S^{k}$. Thus, $T U Y$ is diffeomorphic to $\phi_{1}\left(\mathcal{U}_{1} \cap Y\right) \times S^{k}$.

Now, to lighten notations, let us suppose that $T X=\phi_{1}\left(\mathcal{U}_{1}\right) \times \mathbb{R}^{k+1}$ and $T Y=$ 
$\phi_{1}\left(\mathcal{U}_{1} \cap Y\right) \times \mathbb{R}^{k+1}$.

We also know that

$$
T_{(x, \widehat{V}(x))} G_{-\nabla \widehat{\rho}}=G_{-D \nabla \widehat{\rho}(x)}=\left\{(u,-D \nabla \widehat{\rho}(x)(u)) \mid u \in T_{x} Y\right\}
$$

and

$$
T_{(x, \widehat{V}(x))} G_{\widehat{V}}=G_{D \widehat{V}(x)}=\left\{(u, D \widehat{V}(x)(u)) \mid u \in T_{x} Y\right\} .
$$

These sets are both of dimension $k$.

As the vector field $\nabla \widehat{\rho}$ is unit and radial on $Y$ and so that $-\nabla \hat{\rho}$ points in each point of $\phi_{1}\left(\mathcal{U}_{1}\right) \cap Y$ toward $\phi_{1}(Z)$, we can write in local coordinates that $-\nabla \hat{\rho}$ is constant equal to $(0, \ldots, 0,1) \in \mathbb{R}^{k+1}$ pointing toward $\mathcal{U}_{1} \cap\left\{x_{k+1} \geq 0\right\}$.

Then we find a basis of $T_{(x, \widehat{V}(x))} G_{-\nabla \widehat{\rho}}$ :

$$
\left\langle\left(e_{1},-D \nabla \widehat{\rho}(x)\left(e_{1}\right)\right), \ldots,\left(e_{k},-D \nabla \widehat{\rho}(x)\left(e_{k}\right)\right)\right\rangle \text {. }
$$

But $D \nabla \widehat{\rho}(x)\left(e_{i}\right)=0$ for all $i \in\{1, \ldots, k\}$ so the basis is finally

$$
\left\langle\left(e_{1}, 0\right), \ldots,\left(e_{k}, 0\right)\right\rangle, \quad \text { with }\left(e_{i}, 0\right) \in \mathbb{R}^{k} \times\{0\}^{k} .
$$

Moreover, the sphere $S^{k}$ is locally diffeomorphic to an open set of $\mathbb{R}^{k}$ (by the projection $\left(x_{1}, \ldots, x_{k+1}\right) \in S^{k} \mapsto\left(x_{1}, \ldots, x_{k}\right)$ at $\left.(0, \ldots, 0,1)\right)$ and thus,

$$
T_{(x, \widehat{V}(x))} T U Y \cong \mathbb{R}^{k} \times \mathbb{R}^{k}
$$

where $\cong$ means "diffeomorphic to".

Then we choose a basis for $T_{(x, \widehat{V}(x))} T U Y$ :

$$
\mathcal{B}=\left\langle\left(e_{1}, 0\right), \ldots,\left(e_{k}, 0\right),\left(0, e_{1}\right), \ldots,\left(0, e_{k}\right)\right\rangle,
$$

where $\left(e_{1}, \ldots, e_{k}\right)$ is the canonical basis positively oriented of $\mathbb{R}^{k}$ and for all $i \in\{1, \ldots, k\}$, $\left(e_{i}, 0\right) \in \mathbb{R}^{k} \times\{0\}^{k}$.

Regarding $T_{(x, \widehat{V}(x))} G_{\widehat{V}}$, we have

$$
D \widehat{V}(x): T_{x} Y \longrightarrow \mathbb{R}^{k}
$$

so the image of $D \widehat{V}(x)$ is also in $\mathbb{R}^{k}$. In the same way as previously, a basis of $T_{(x, \widehat{V}(x))} G_{\widehat{V}}$ is of the form

$$
\left\langle\left(e_{1}, D \widehat{V}(x)\left(e_{1}\right)\right), \ldots,\left(e_{k}, D \widehat{V}(x)\left(e_{k}\right)\right)\right\rangle .
$$

Thus we obtained a basis of $T_{(x, \widehat{V}(x))} G_{-\nabla \widehat{\rho}} \times T_{(x, \widehat{V}(x))} G_{\widehat{V}}$ :

$$
\mathcal{B}^{\prime}=\left\langle\left(e_{1}, 0\right), \ldots,\left(e_{k}, 0\right),\left(e_{1}, D \widehat{V}(x)\left(e_{1}\right)\right), \ldots,\left(e_{k}, D \widehat{V}(x)\left(e_{k}\right)\right)\right\rangle .
$$


Let us compute $D \widehat{V}(x)\left(e_{i}\right)$ for all $i \in\{1, \ldots, k\}$.

We know that $\widehat{V}(x)=(0, \ldots, 0,1) \in S^{k}$ so in local coordinates, we have

$$
\widehat{V}(x)=\left(\widehat{V}_{1}(x), \ldots, \widehat{V}_{k}(x)\right)=\left(\frac{V_{1}(x)}{\|V(x)\|}, \ldots, \frac{V_{k}(x)}{\|V(x)\|}\right) .
$$

Then for all $i, j \in\{1, \ldots, k\}$, we have

$$
\frac{\partial \widehat{V}_{i}}{\partial x_{j}}(x)=\frac{\frac{\partial V_{i}}{\partial x_{j}}(x)\|V(x)\|-\frac{\partial\|V\|}{\partial x_{j}}(x) V_{i}(x)}{\|V(x)\|^{2}} .
$$

But $V_{i}(x)=0$ for all $i \in\{1, \ldots, k\}$, thus

$$
\frac{\partial \widehat{V}_{i}}{\partial x_{j}}(x)=\frac{1}{\|V(x)\|} \frac{\partial V_{i}}{\partial x_{j}}(x) .
$$

We finally have

$$
D \widehat{V}(x)\left(e_{i}\right)=\frac{1}{\|V(x)\|}\left(\sum_{j=1}^{k} \frac{\partial V_{1}}{\partial x_{j}}(x) e_{i}^{j}, \ldots, \sum_{j=1}^{k} \frac{\partial V_{k}}{\partial x_{j}}(x) e_{i}^{j}\right),
$$

where we wrote $e_{i}=\left(e_{i}^{1}, \ldots, e_{i}^{k}\right)$ the $k$ components of $e_{i}$. We also know that $e_{i}^{j}=0$ for all $i \neq j$ and $e_{i}^{i}=1$.

We set

$$
\begin{aligned}
& A:=\left[\begin{array}{lll}
D \widehat{V}(x)\left(e_{1}\right) & \cdots & D \widehat{V}(x)\left(e_{k}\right)
\end{array}\right] \\
& =\frac{1}{\|V(x)\|}\left[\begin{array}{ccc}
\frac{\partial V_{1}}{\partial x_{1}}(x) & \cdots & \frac{\partial V_{1}}{\partial x_{k}}(x) \\
\vdots & & \vdots \\
\frac{\partial V_{k}}{\partial x_{1}}(x) & \cdots & \frac{\partial V_{k}}{\partial x_{k}}(x)
\end{array}\right] \times \underbrace{\left[\begin{array}{ccc}
e_{1}^{1} & \cdots & e_{k}^{1} \\
\vdots & & \vdots \\
e_{1}^{k} & \cdots & e_{k}^{k}
\end{array}\right]}_{:=I d_{\mathbb{R} k}}=\frac{1}{\|V(x)\|} D V_{\mid Y}(x) .
\end{aligned}
$$

Now we can write the change of basis $P$ from $\mathcal{B}^{\prime}$ to $\mathcal{B}$ :

$$
P=\left[\begin{array}{c|c}
I d_{\mathbb{R}^{k}} & I d_{\mathbb{R}^{k}} \\
\hline(0) & A
\end{array}\right],
$$

then

$$
\operatorname{det} P=\operatorname{det} A=\frac{1}{\|V(x)\|} \operatorname{det} D V_{\mid Y}(x) .
$$

Even if we consider a perturbation $\widetilde{V}$ of $V$ which only admits nondegenerate zeros on $Y$, we suppose, to simplify, that it is already the case for $V$. Thus, as $x$ is a nondegenerate zero of $V$, we have $\operatorname{det} D V_{\mid Y}(x) \neq 0$. We finally have

$$
\operatorname{sign}(\operatorname{det} P)=\operatorname{sign}\left(\operatorname{det} D V_{\mid Y}(x)\right) .
$$


Thus we have proved that for each coincidence point $x$ of $-\nabla \widehat{\rho}$ and $\widehat{V}$, which gives us an intersection point $(x, \widehat{V}(x)) \in T U Y$ of $G_{-\nabla \widehat{\rho}}$ and $G_{\widehat{V}}$, we have

$$
\operatorname{sign}\left(\operatorname{det} D V_{\mid Y}(x)\right)=\operatorname{sign}(\operatorname{det} P),
$$

where $\operatorname{sign}(\operatorname{det} P)$ is just $\operatorname{or}(x)$.

Thus,

$$
\sum_{x \in C(-\nabla \widehat{\rho}, \widehat{V})} \operatorname{or}(x)=\sum_{x \text { inward zero of } V_{\mid Y}} \operatorname{sign}\left(\operatorname{det} D V_{\mid Y}(x)\right) .
$$

But

$$
\sum_{x \text { inward zero of } V_{\mid Y}} \operatorname{sign}\left(\operatorname{det} D V_{\mid Y}(x)\right)=\sum_{x \text { inward zero of } V_{\mid Y}} i n d_{P H}\left(V_{\mid Y}, x\right),
$$

and we proved that

$$
\sum_{x \text { inward zero of } V_{\mid Y}} \operatorname{ind}_{P H}\left(V_{\mid Y}, x\right)=1-i n d_{\text {rad }}(V, 0) .
$$

Last we have, by definition,

$$
I\left(G_{-\nabla \widehat{\rho}}, G_{\widehat{V}}\right)=\sum_{x \in C(-\nabla \widehat{\rho}, \widehat{V})} \operatorname{or}(x) .
$$

This finally gives us

$$
I\left(G_{-\nabla \widehat{\rho}}, G_{\widehat{V}}\right)=1-i n d_{r a d}(V, 0) .
$$

\section{REFERENCES}

[1] M. Aguilar, J.A. Seade, and A. Verjovsky. Indices of vector fields and topological invariants or real analytic singularities. J. Reine Angew. Math, 504:159-176, 1998.

[2] V.I. Arnol'd. Indexes of singular points of 1-forms on manifolds with boundary, convolutions of invariants of groups generated by reflections, and singular projections of smooth surfaces. Uspekhi Mat. Nauk, 34:3-38, 1979.

[3] J.-P. Brasselet, J. Seade, and T. Suwa. Vector fields on singular varieties. Springer, 2009.

[4] N. Dutertre. Radial index and Poincaré-Hopf index of 1-forms on semi-analytic sets. Math. Proc. Cambridge Phil. Soc., 148:297-330, 2010.

[5] W. Ebeling and S.M. Gusein-Zade. On the index of a vector field at an isolated singularity. Fields Inst. Commun., 24, 1999.

[6] W. Ebeling and S.M. Gusein-Zade. Radial index and Euler obstruction of a 1-form on a singular variety. Geom. Dedicata, 113:231-241, 2005.

[7] W. Ebeling, S.M. Gusein-Zade, and J. Seade. Homological index for 1-forms and a Milnor number for isolated singularities. Internat. J. Math., 15:895-905, 2004.

[8] V. Guillemin and A. Pollack. Differential topology. Prentice-Hall, 1974.

[9] G.M. Khimshiashvili. On the local degree of a smooth map. Soobshch. Akad. Nauk Gruz., 85:309311, 1977.

[10] H. King and D. Trotman. Poincaré-hopf theorems on singular spaces. Proc. Lond. Math. Soc., 108:682-703, 2014. 
[11] M.-H. Schwartz. Classes caractéristiques définies par une stratification d'une variété analytique complexe. C.R. Acad. Sci. Paris Sér. I Math., 260:3262-3264,3535-3537, 1965.

[12] M.-H. Schwartz. Champs radiaux sur une stratification analytique, volume 39. Hermann, 1991.

[13] J. Seade and T. Suwa. A residue formula for the index of a holomorphic flow. Math. Ann., 304:621-634, 1996.

Aix-Marseille Université, CnRs, Centre Gilles-Gaton Granger, Aix-en-Provence, FRANCE

E-mail address: julie.lapebie@univ-amu.fr 